\title{
LMI Approach to Stability Analysis of Cohen-Grossberg Neural Networks with $p$-Laplace Diffusion
}

\author{
Xiongrui Wang, ${ }^{1,2}$ Ruofeng Rao, ${ }^{1,2}$ and Shouming Zhong ${ }^{2,3}$ \\ ${ }^{1}$ Department of Mathematics, Yibin University, Yibin 644007, China \\ ${ }^{2}$ Institute of Mathematics, Yibin University, Yibin 644007, China \\ ${ }^{3}$ College of Applied Mathematics, University of Electronic Science and Technology of China, \\ Chengdu 610054, China \\ Correspondence should be addressed to Ruofeng Rao, ruofengrao@163.com
}

Received 1 August 2012; Revised 24 October 2012; Accepted 12 November 2012

Academic Editor: Zhilong L. Huang

Copyright (C) 2012 Xiongrui Wang et al. This is an open access article distributed under the Creative Commons Attribution License, which permits unrestricted use, distribution, and reproduction in any medium, provided the original work is properly cited.

\begin{abstract}
The nonlinear $p$-Laplace diffusion $(p>1)$ was considered in the Cohen-Grossberg neural network (CGNN), and a new linear matrix inequalities (LMI) criterion is obtained, which ensures the equilibrium of CGNN is stochastically exponentially stable. Note that, if $p=2, p$-Laplace diffusion is just the conventional Laplace diffusion in many previous literatures. And it is worth mentioning that even if $p=2$, the new criterion improves some recent ones due to computational efficiency. In addition, the resulting criterion has advantages over some previous ones in that both the impulsive assumption and diffusion simulation are more natural than those of some recent literatures.
\end{abstract}

\section{Introduction and Preparation}

It is well known that Cohen-Grossbeg neural network (CGNN) was proposed by Cohen and Grossberg [1] in 1983. Since then there have been a lot of interested results obtained in many literatures (see [2-9]) due to its general applications, such as pattern recognition, image and signal processing, optimization automatic control, and artificial intelligence. Usually, there exist the impulsive effect and time-varying delays phenomenon in various neural networks $[3,5-7,10-14]$. Besides, diffusion effects cannot be avoided in the neural networks when electrons are moving in asymmetric electromagnetic fields [15-18]. However, diffusion disturbance was always simulated simply by linear Laplace diffusion [15-18]. Few papers 
involved the nonlinear reaction-diffusion [19]. So in this paper, we investigate the stability of the following stochastic CGNN with nonlinear $p$-Laplace diffusion $(p>1)$ :

$$
\begin{aligned}
& d u(t, x)=\{ \nabla \cdot\left(\tilde{D}(t, x, u) \circ \nabla_{p} u\right)-A(u(t, x)) \\
& \times {[B(u(t, x))-C f(u(t, x))+D g(u(t-\tau(t), x))]\} d t } \\
&+ \sigma(u(t, x)) d w(t), \quad t \in\left[t_{k}, t_{k+1}\right) \\
& u\left(t_{k}^{+}, x\right)=M u\left(t^{-}, x\right), \quad t=t_{k} \\
& u\left(t_{0}+\theta, x\right)=\varphi(\theta, x), \quad(\theta, x) \in[-\tau, 0] \times \partial \Omega \\
& \frac{\partial u_{i}(t, x)}{\partial \mathcal{v}}=0, \quad(t, x) \in[-\tau,+\infty) \times \partial \Omega, \quad i=1,2, \ldots, n,
\end{aligned}
$$

where $\Omega$ is a bounded subset in $R^{m}$ with smooth boundary $\partial \Omega$, and $\partial u_{i}(t, x) / \partial v=$ $\left(\left(\partial u_{i}(t, x) / \partial x_{1}\right),\left(\partial u_{i}(t, x) / \partial x_{2}\right), \ldots,\left(\partial u_{i}(t, x) / \partial x_{m}\right)\right)^{T}$ denotes the outward normal derivative on $\partial \Omega$.

Remark 1.1. If $p=2$, system (1.1) was studied by [5] though there is a little difference between Dirichlet boundary condition and Neumann boundary condition. However, our impulsive assumption $u\left(t_{k}^{+}, x\right)=M u\left(t_{k}^{-}, x\right)$ is more natural than that of [5], which will result in some difference in methods.

Here, $M$ is a diagonal matrix, $\Omega \in R^{m}$ is a bounded compact set with smooth boundary, $u=\left(u_{1}, u_{2}, \ldots, u_{n}\right)^{T} \in R^{n}$, and $w(t)=\left(w_{1}(t), w_{2}(t), \ldots, w_{n}(t)\right)^{T}$ is a $n$-dimensional Brownian motion defined on a complete probability space $(\Omega, \mathcal{F}, P)$ with the natural filtration $\left\{\mathcal{F}_{t}\right\}_{t \geq 0}$ generated by the process $\{w(s): 0 \leq s \leq t\}$. We associate $\Omega$ with the canonical space generated by all $\left\{w_{i}(t)\right\}$ and denoted by $\mathcal{F}$ the associated $\sigma$-algebra generated by $w(t)$ with the probability measure $p$. $A(u(t, x))$ presents an amplification function, $B(u(t, x))$ is an appropriately behavior function, and $f$ and $g$ denote the activation function. $\tau(t)(0 \leq \tau(t) \leq$ $\tau)$ corresponds to the transmission delays at time, and $t_{k}$ is called the impulsive moment with $0<t_{1}<t_{2}<\cdots<t_{k}<\cdots$ and $\lim _{k \rightarrow \infty} t_{k}=+\infty$. We always assume $u\left(t_{k}^{+}, x\right)=$ $u\left(t_{k}, x\right) . \nabla_{p} u=\left(\nabla_{p} u_{1}, \ldots, \nabla_{p} u_{n}\right)^{T}, \nabla_{p} u_{i}=\left(\left|\nabla u_{i}\right|^{p-2}\left(\partial u_{i} / \partial x_{1}\right), \ldots,\left|\nabla u_{i}\right|^{p-2}\left(\partial u_{i} / \partial x_{m}\right)\right)^{T}, \tilde{D} \circ$ $\nabla_{p} u=\left(\tilde{D}_{i k}\left|\nabla u_{i}\right|^{p-2}\left(\partial u_{i} / \partial x_{k}\right)\right)_{n \times m}$ is Hadamard product of matrix $\tilde{D}$ and $\nabla_{p} u$ [20]. Here, the diffusion parameters matrix $\tilde{D}(t, x, u)$ is denoted simply as $\tilde{D}=\left(\tilde{D}_{i k}\right)_{n \times m}$. Let $Y_{i}=$ $\left(y_{i 1}, \ldots, y_{i m}\right)^{T}, i=1,2, \ldots, n$, and matrix $\mathbb{Y}=\left(Y_{1}, \ldots, Y_{n}\right)^{T}$, and we denote $\nabla \cdot Y_{i}=$ $\sum_{k=1}^{m} \partial y_{i k} / \partial x_{k}, \nabla \cdot \mathbb{Y}=\left(\nabla \cdot Y_{1}, \nabla \cdot Y_{2}, \ldots, \nabla \cdot Y_{n}\right)^{T}$. Particularly, $\nabla_{p} u=\nabla u$ for the case of $p=2$.

Remark 1.2. Diffusion effects always occur in the neural networks when electrons are moving in asymmetric electromagnetic fields [15-18], and diffusion behavior is so complicated that it cannot always be simulated by linear Laplace diffusion. So in this paper, the nonlinear $p$-Laplace diffusion is considered in System (1.1). 
Assume, in addition, the following.

(H1) $A(u(t, x))$ is a bounded, positive, and continuous diagonal matrix, that is, there exist two positive diagonal matrices $\underline{A}$ and $\bar{A}$ such that $0<\underline{A} \leq A(u(t, x)) \leq \bar{A}$.

(H2) $B(u(t, x))=\left(b_{1}\left(u_{1}(t, x)\right), b_{2}\left(u_{2}(t, x)\right), \ldots, b_{n}\left(u_{n}(t, x)\right)\right)^{T}$ such that there exists a positive diagonal matrix $B=\operatorname{diag}\left(B_{1}, B_{2}, \ldots, B_{n}\right)$ satisfying $\left(b_{i}\left(u_{i}(t, x)\right) / u_{i}(t, x)\right) \geq$ $B_{i}$ for all $i$.

(H3) There exist two positive diagonal matrices $F=\operatorname{diag}\left(F_{1}, F_{2}, \ldots, F_{n}\right)$ and $G=$ $\operatorname{diag}\left(G_{1}, G_{2}, \ldots, G_{n}\right)$ such that

$$
0 \leq \frac{f_{i}(r)}{r} \leq F_{i}, \quad 0 \leq \frac{g_{i}(r)}{r} \leq G_{i}, \quad \forall i
$$

(H4) The null solution is the equilibrium point of system (1.1), that is, the following conditions hold:

$$
B(0)-C f(0)-D g(0)=0, \quad \operatorname{trace}\left[\sigma^{T}(u(t, x)) \sigma(u(t, x))\right] \leq u^{T}(t, x) Q u(t, x),
$$

where the symmetrical matrix $Q>0$.

For convenience's sake, we introduce some standard notations

(i) $L^{2}(R)(R \times \Omega)$ : the space of real Lebesgue measurable functions of $R \times \Omega$, it is a Banach space for the 2-norm $\|u(t)\|_{2}=\left(\sum_{i=1}^{n}\left\|u_{i}(t)\right\|\right)^{1 / 2}$ with $\left\|u_{i}(t)\right\|=$ $\left(\int_{\Omega}\left|u_{i}(t, x)\right|^{2} d x\right)^{1 / 2}$, where $\left|u_{i}(t, x)\right|$ is Euclid norm.

(ii) $L_{F_{0}}^{2}\left([-\tau, 0] \times \Omega ; R^{n}\right)$ : the family of all $F_{0}$-measurable $C\left([-\tau, 0] \times \Omega ; R^{n}-\right)$ value random variable $\xi=\{\xi(\theta, x):-\tau \leq \theta \leq 0, x \in \Omega\}$ such that $\sup _{-\tau \leq \theta \leq 0} E\|\xi(\theta)\|_{2}^{2}<\infty$, where $E\{\cdot\}$ stands for the mathematical expectation operator with respect to the given probability measure $p$.

(iii) $Q=\left(q_{i j}\right)_{n \times n}>0(<0)$ : a positive (negative) definite symmetrical matrix, that is, $y^{T} Q y>0(<0)$ for any $0 \neq y \in R^{n}$.

(iv) $Q=\left(q_{i j}\right)_{n \times n} \geq 0(\leq 0)$ : a semipositive (semi-negative) definite symmetrical matrix, that is, $y^{T} Q y \geq 0(\leq 0)$ for any $y \in R^{n}$.

(v) $Q \geq \widetilde{Q}(Q \leq \widetilde{Q})$ : this means $Q-\widetilde{Q}$ is a semi-positive (semi-negative) symmetrical definite matrix.

(vi) $Q>\widetilde{Q}(Q<\tilde{Q})$ : this means $Q-\widetilde{Q}$ is a positive (negative) symmetrical definite matrix.

(vii) $\lambda_{\max }(\Phi), \lambda_{\min }(\Phi)$ denotes the largest and smallest eigenvalue of symmetrical matrix $\Phi$, respectively.

(viii) $I$ : identity matrix with compatible dimension.

(ix) Denote $|C|=\left(\left|c_{i j}\right|\right)_{n \times n}$ for any matrix $C_{n \times n} ;|u(t, x)|=\left(\left|u_{1}\right|,\left|u_{2}\right|, \ldots,\left|u_{n}\right|\right)$ for any $u \in R^{n}$.

Let $u(t, x ; \varphi)$ denote the state trajectory from the initial data $u\left(t_{0}+\theta, x ; \varphi\right)=\varphi(\theta, x)$ on $-\tau \leq \theta \leq 0$ in $L_{F_{0}}^{2}\left([-\tau, 0] \times \Omega ; R^{n}\right)$. 
Definition 1.3. The null solution of impulsive system (2.2) is globally stochastically exponentially stable in the mean square if for every $\varphi \in L_{F_{0}}^{2}\left([-\tau, 0] \times \Omega ; R^{n}\right)$, there exists scalars $\beta>0$ and $\gamma>0$ such that

$$
E\left(\|u(t, \varphi)\|_{2}^{2}\right) \leq r e^{-\beta t} \sup _{-\tau \leq \theta \leq 0} E\left(\|\varphi(\theta)\|_{2}^{2}\right) .
$$

Lemma 1.4 (see [11]). Let $U, P$ be any matrices, $\varepsilon>0$ is a positive number and matrix $H=H^{T}>0$, then

$$
P^{T} U+U^{T} P \leq \varepsilon P^{T} H P+\varepsilon^{-1} U^{T} H^{-1} U
$$

Lemma 1.5 (Schur complement [3]). The LMI

$$
\left(\begin{array}{cc}
Q(t) & S(t) \\
S^{T}(t) & R(t)
\end{array}\right)>0
$$

where matrix $S(t)$ and symmetrical matrices $Q(t)$ and $R(t)$ depend on $t$, is equivalent to any one of the following conditions:

$\left(\mathrm{L}_{1}\right) R(t)>0, Q(t)-S(t) R^{-1}(t) S^{T}(t)>0 ;$

$\left(\mathrm{L}_{2}\right) Q(t)>0, R(t)-S^{T}(t) Q^{-1}(t) S^{T}(t)>0$.

Lemma 1.6 (see [21]). Consider the following differential inequality:

$$
\begin{gathered}
D^{+} v(t) \leq-a v(t)+b[v(t)]_{\tau^{\prime}} \quad t \neq t_{k} \\
v\left(t_{k}\right) \leq a_{k} v\left(t_{k}^{-}\right)+b_{k}\left[v\left(t_{k}^{-}\right)\right]_{\tau^{\prime}}
\end{gathered}
$$

where $v(t) \geq 0,\left[v\left(t_{k}\right)\right]_{\tau}=\sup _{t-\tau \leq s \leq t} v(s),\left[v\left(t_{k}^{-}\right)\right]_{\tau}=\sup _{t-\tau \leq s \leq t} v(s)$ and $v(t)$ is continuous except $t_{k}, k=1,2, \ldots$, where it has jump discontinuities. The sequence $t_{k}$ satisfies $0=t_{0}<t_{1}<t_{2}<\cdots<$ $t_{k}<t_{k+1}<\cdots$, and $\lim _{k \rightarrow \infty} t_{k}=\infty$. Suppose that

(1) $a>b \geq 0$;

(2) $t_{k}-t_{k-1}>\delta \tau$, where $\delta>1$, and there exist constants $\gamma>0, M>0$ such that

$$
\rho_{1} \rho_{2} \cdots \rho_{k+1} \leq M e^{\gamma t_{k}}
$$

where $\rho_{i}=\max \left\{1, a_{i}+b_{i} e^{\lambda \tau}\right\}, \lambda>0$ is the unique solution of equation $\lambda=a-b e^{\lambda \tau}$ then

$$
v(t) \leq M[v(0)]_{\tau} e^{-(\lambda-\gamma) t} .
$$

In addition, if $\theta=\sup _{k \in Z}\left\{1, a_{k}+b_{k} e^{\lambda \tau}\right\}$, then

$$
v(t)=\theta[v(0)]_{\tau} e^{-\left(\lambda-\left(\ln \left(\theta e^{\lambda \tau}\right) / \delta \tau\right)\right) t}, \quad t \geq 0
$$

Computer simulation is shown in Figures 1, 2, 3, and 4. 


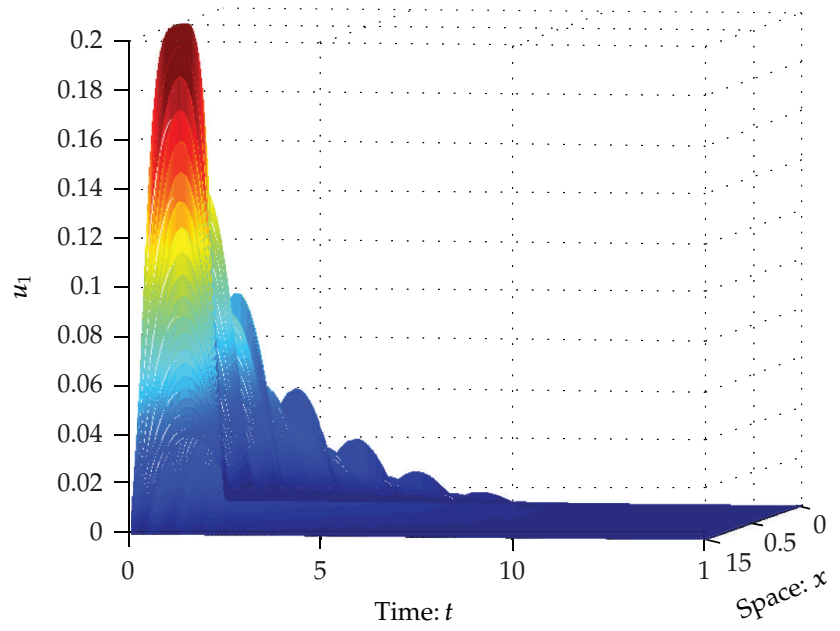

Figure 1: Computer simulation of the state $u_{1}(t, x)$.

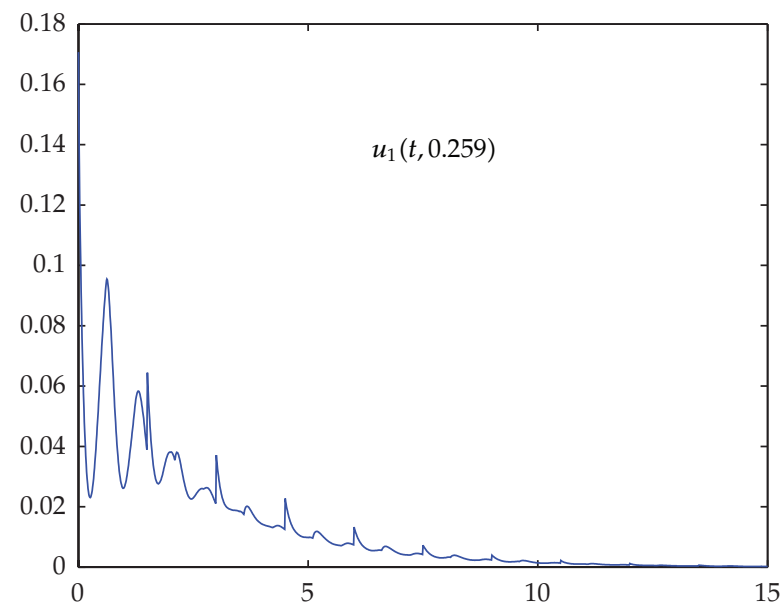

Figure 2: Sectional curve of the state variable $u_{1}(t, x)$.

\section{Main Results}

Theorem 2.1. If assumptions (H1)-(H4) hold, in addition, the following conditions are satisfied:

(C1) there exists diagonal matrices $P_{1}=\operatorname{diag}\left(p_{11}, p_{12}, \ldots, p_{1 n}\right)>0$ and $P_{2}>0$ such that

$$
\left(\begin{array}{ccc}
-2 P_{1} \underline{A} B+F^{2}+P_{1} Q+P_{2} & P_{1} \bar{A}|C| & P_{1} \bar{A}|D| \\
\left|C^{T}\right| \bar{A} P_{1} & -I & 0 \\
\left|D^{T}\right| \bar{A} P_{1} & 0 & -I
\end{array}\right)<0
$$

(C2) $\min \left\{\left(\lambda_{\min } \Theta / \lambda_{\max } P_{1}\right),(1-\mu)\right\}>\left(\lambda_{\max } G^{2} / \lambda_{\min } P_{2}\right) \geq 0$, where $\Theta=2 P_{1} \underline{A} B-$ $P_{1} \bar{A}|C||C|^{T} \bar{A} P_{1}-P_{1} \bar{A}|D \| D|^{T} \bar{A} P_{1}-F^{2}-P_{1} Q-P_{2}$, where $\tau^{\prime}(t) \leq \mu<1$ for all $t$; 


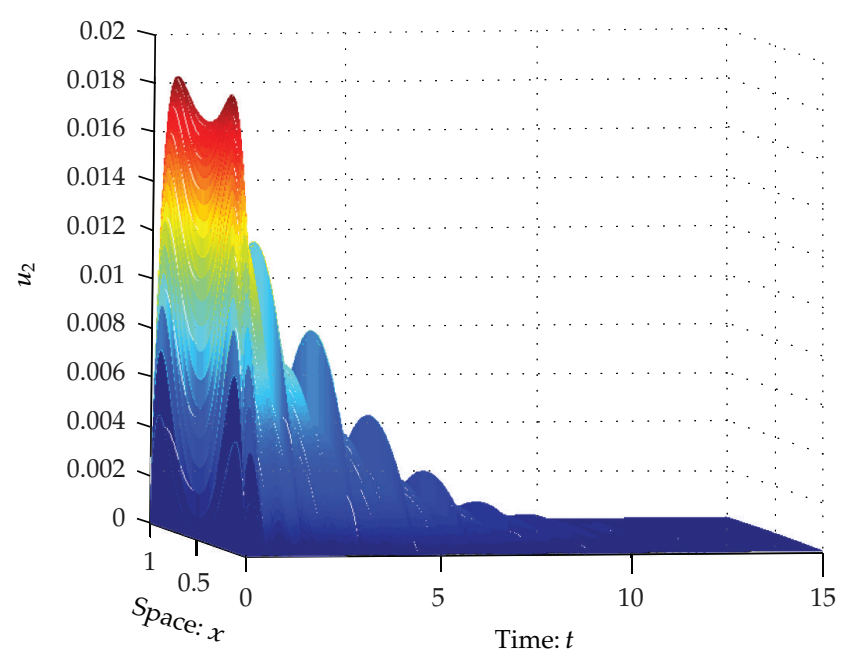

Figure 3: Computer simulation of the state $u_{2}(t, x)$.

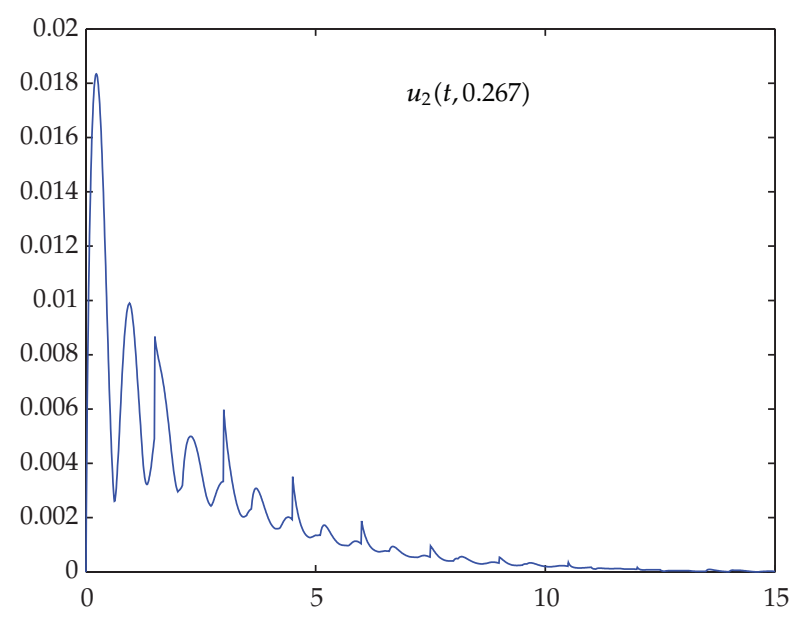

Figure 4: Sectional curve of the state variable $u_{2}(t, x)$.

(C3) there exists a constant $\delta>1$ such that $\inf _{k \in Z}\left(t_{k}-t_{k-1}\right)>\delta \tau, \delta^{2} \tau>\ln \left(\rho e^{\lambda \tau}\right)$ and $\lambda-\left(\ln \left(\rho e^{\lambda \tau}\right) / \delta \tau\right)>0$, where $\lambda>0$ is the unique solution of the equation $\lambda=a-b e^{\lambda \tau}$, and $\rho=\max \left\{1,\left(\lambda_{\max }\left(M P_{1} M\right) / \lambda_{\min } P_{1}\right)+e^{\lambda \tau}\right\}, a=\min \left\{\left(\lambda_{\min } \Theta / \lambda_{\max } P_{1}\right),(1-\mu)\right\}, b=$ $\lambda_{\max } G^{2} / \lambda_{\min } P_{2}$, then the null solution of system (1.1) is stochastically exponentially stable with convergence rate $(1 / 2)\left(\lambda-\left(\ln \left(\rho e^{\lambda \tau}\right) / \delta \tau\right)\right)$.

Proof. First, we can get by Guass formula (see [20, Lemma 2.3])

$$
\begin{aligned}
& \int_{\Omega} u^{T} P_{1}\left(\nabla \cdot\left(\tilde{D}(t, x, u) \circ \nabla_{p} u\right) d x\right. \\
& \quad=\int_{\Omega} u^{T} P_{1}\left(\sum_{k=1}^{m} \frac{\partial}{\partial x_{k}}\left(\widetilde{D}_{1 k}\left|\nabla u_{1}\right|^{p-2} \frac{\partial u_{1}}{\partial x_{k}}\right), \ldots, \sum_{k=1}^{m} \frac{\partial}{\partial x_{k}}\left(\widetilde{D}_{n k}\left|\nabla u_{n}\right|^{p-2} \frac{\partial u_{n}}{\partial x_{k}}\right)\right)^{T} d x
\end{aligned}
$$


Journal of Applied Mathematics

$$
\begin{aligned}
& =\int_{\Omega} \sum_{j=1}^{n} p_{1 j} u_{j} \sum_{k=1}^{m} \frac{\partial}{\partial x_{k}}\left(\tilde{D}_{j k}\left|\nabla u_{j}\right|^{p-2} \frac{\partial u_{j}}{\partial x_{k}}\right) d x \\
& =-\sum_{k=1}^{m} \sum_{j=1}^{n} \int_{\Omega} p_{1 j} \tilde{D}_{j k}\left|\nabla u_{j}\right|^{p-2}\left(\frac{\partial u_{j}}{\partial x_{k}}\right)^{2} d x .
\end{aligned}
$$

Construct the Lyapunov functional as follows:

$$
V=V_{1}+V_{2}
$$

where

$$
\begin{gathered}
V_{1}=\int_{\Omega} u^{T}(t, x) P_{1} u(t, x) d x=\int_{\Omega}\left|u^{T}(t, x)\right| P_{1}|u(t, x)| d x \\
V_{2}=\int_{\Omega} \int_{t-\tau(t)}^{t} u^{T}(s, x) P_{2} u(s, x) d s d x .
\end{gathered}
$$

Then

$$
\begin{aligned}
L V_{1}= & \int_{\Omega}-\sum_{k=1}^{m} \sum_{j=1}^{n} 2 p_{1 j} \widetilde{D}_{j k}\left|\nabla u_{j}\right|^{p-2}\left(\frac{\partial u_{j}}{\partial x_{k}}\right)^{2} d x \\
& -2 \int_{\Omega} u^{T}(t, x) P_{1} A(u(t, x)) B(u(t, x)) d x \\
& +2 \int_{\Omega} u^{T}(t, x) P_{1} A(u(t, x)) C f(u(t, x)) d x \\
& +2 \int_{\Omega} u^{T}(t, x) P_{1} A(u(t, x)) D g(u(t-\tau(t), x)) d x \\
& +\int_{\Omega} \operatorname{trace}\left[\sigma^{T}(u(t, x)) P_{1} \sigma(u(t, x))\right] d x \\
\leq & -2 \int_{\Omega}\left|u^{T}(t, x)\right| P_{1} \underline{A} B|u(t, x)| d x \\
& +\int_{\Omega}\left[\left|u^{T}(t, x)\right| P_{1} \bar{A}|C \| C|^{T} \bar{A} P_{1}|u(t, x)|+\left|f^{T}(u(t, x))\right||f(u(t, x))|\right] d x \\
& +\int_{\Omega}\left[\left|u^{T}(t, x)\right| P_{1} \bar{A}|D \| D|^{T} \bar{A} P|u(t, x)|+g^{T}(u(t-\tau(t), x)) g(u(t-\tau(t), x))\right] d x \\
& +\int_{\Omega} u^{T}(t, x) P_{1} Q u(t, x) d x
\end{aligned}
$$




$$
\begin{aligned}
\leq & -\int_{\Omega}\left|u^{T}(t, x)\right|\left[2 P_{1} \underline{A} B-P_{1} \bar{A}|C||C|^{T} \bar{A} P_{1}-P_{1} \bar{A}|D||D|^{T} \bar{A} P_{1}-F^{2}-P_{1} Q\right]|u(t, x)| d x \\
& +\int_{\Omega} u^{T}(t-\tau(t), x) G^{2} u(t-\tau(t), x) d x \\
L V_{2}= & \int_{\Omega}\left[u^{T}(t, x) P_{2} u(t, x)-\left(1-\tau^{\prime}(t)\right) u^{T}(t-\tau(t)) P_{2} u(t-\tau(t))\right] d x \\
\leq & \int_{\Omega}\left|u^{T}(t, x)\right| P_{2}|u(t, x)| d x+(\mu-1) \int_{\Omega} u^{T}(t-\tau(t)) P_{2} u(t-\tau(t)) d x .
\end{aligned}
$$

And then we have

$$
L V \leq-\int_{\Omega}\left|u^{T}(t, x)\right| \Theta|u(t, x)| d x+\int_{\Omega} u^{T}(t-\tau(t), x)\left[G^{2}+(\mu-1) P_{2}\right] u(t-\tau(t), x) d x .
$$

Next, we use the method similar as that of [22]. Since $u(t, x)$ is the solution of system, and $V(u(t, x)) \in C^{2}\left[R^{m}, R^{+}\right]$for all $t$, we can get by Itô formula

$$
V(t)=V\left(t_{k}\right)+\int_{t_{k}}^{t} L V(u(s, x)) d s+\int_{t_{k}}^{t} \frac{\partial V}{\partial u} \sigma(u(s, x)) d w(s) .
$$

Then we have

$$
E V(u(t, x))=E V\left(u\left(t_{k}, x\right)\right)+\int_{t_{k}}^{t} E L V(s, x) d s, \quad t \in\left[t_{k}, t_{k+1}\right)
$$

Thus, for small enough $\Delta t>0$, we have

$$
E V(u(t+\Delta t, x))=E V\left(u\left(t_{k}, x\right)\right)+\int_{t_{k}}^{t+\Delta t} E L V(s, x) d s, \quad t \in\left[t_{k}, t_{k+1}\right)
$$

and then

$$
\begin{aligned}
& E V(u(t+\Delta t, x))-E V(u(t, x)) \\
& =\int_{t}^{t+\Delta t} E L V(s, x) d s \\
& \leq E \int_{t}^{t+\Delta t}\left[-\int_{\Omega}\left|u^{T}(s, x)\right| \Theta|u(s, x)| d x\right. \\
& \left.\quad+\int_{\Omega} u^{T}(s-\tau(s), x)\left[G^{2}+(\mu-1) P_{2}\right] u(s-\tau(s), x) d x\right] d s, \quad t \in\left[t_{k}, t_{k+1}\right) .
\end{aligned}
$$


Since

$$
\begin{aligned}
& \int_{\Omega}\left|u^{T}(t, x)\right| \Theta|u(t, x)| d x+\int_{\Omega} u^{T}(t-\tau(t), x)(1-\mu) P_{2} u(t-\tau(t), x) d x \\
& \quad \geq \frac{\lambda_{\min } \Theta}{\lambda_{\max } P_{1}} \int_{\Omega}\left|u^{T}(t, x)\right| P_{1}|u(t, x)| d x+(1-\mu) \int_{\Omega} u^{T}(t-\tau(t), x) P_{2} u(t-\tau(t), x) d x \\
& \quad \geq \min \left\{\frac{\lambda_{\min } \Theta}{\lambda_{\max } P_{1}},(1-\mu)\right\} V \\
& \int_{\Omega} u^{T}(t-\tau(t), x) G^{2} u(t-\tau(t), x) d x \leq \frac{\lambda_{\max } G^{2}}{\lambda_{\min } P_{2}} \int_{\Omega} u^{T}(t-\tau(t), x) P_{2} u(t-\tau(t), x) d x,
\end{aligned}
$$

Then,

$$
D^{+} E V(u(t, x)) \leq-\min \left\{\frac{\lambda_{\min } \Theta}{\lambda_{\max } P_{1}},(1-\mu)\right\} E V(t)+\frac{\lambda_{\max } G^{2}}{\lambda_{\min } P_{2}}[E V(t)]_{\tau}, \quad t \in\left[t_{k}, t_{k+1}\right)
$$

Next, we have

$$
\begin{aligned}
V\left(t_{k}\right) & =\int_{\Omega} u^{T}\left(t_{k}, x\right) P_{1} u\left(t_{k}, x\right) d x+\int_{\Omega} u^{T}\left(t_{k}-\tau\left(t_{k}\right), x\right) P_{2} u\left(t_{k}-\tau\left(t_{k}\right), x\right) d x \\
& =\int_{\Omega}\left[u^{T}\left(t_{k}^{-}, x\right) M P_{1} M u\left(t_{k^{\prime}}^{-} x\right) d x+\int_{\Omega} u^{T}\left(t_{k}-\tau\left(t_{k}\right), x\right) P_{2} u\left(t_{k}-\tau\left(t_{k}\right), x\right) d x\right. \\
& \leq \frac{\lambda_{\max }\left(M P_{1} M\right)}{\lambda_{\min } P_{1}} V\left(t_{k}^{-}\right)+\left[V\left(t_{k}^{-}\right)\right]_{\tau} .
\end{aligned}
$$

Now the conditions (C1)-(C3) and Lemma 1.6 deduce

$$
E V(t) \leq \rho[V(0)]_{\tau} e^{-\left(\lambda-\left(\ln \left(\rho e^{\lambda \tau}\right) / \delta \tau\right)\right) t}
$$

or

$$
E\|u(t, x)\|^{2} \leq\left(\rho \frac{\lambda_{\max } P}{\lambda_{\min } P} \sup _{-\tau \leq s \leq 0} E\|\phi(s)\|_{2}^{2}\right) e^{-\left(\lambda-\left(\ln \left(\rho e^{\lambda \tau}\right) / \delta \tau\right)\right) t}
$$

which together with Definition 1.3 implies the accomplishment of the proof.

Remark 2.2. The nonlinear $p$-Laplace diffusion $(p>1)$ brings a great difficulties in judging the stability. However, even if $p=2$, Theorem 2.1 has more computational efficiency than [15, Theorem 3.1] due to LMI criterion. 


\section{Examples}

Consider the following impulsive CGNN:

$$
\begin{gathered}
\left.\left.d u(t, x)=\left\{\begin{array}{c}
\nabla \\
\times\left(\left(\begin{array}{cc}
0.0005 & 0 \\
0 & 0.0005
\end{array}\right) \circ \nabla_{p} u\right)-\left(\begin{array}{cc}
1.65+0.5 \sin u_{1} & 0 \\
0 & 1.58+0.5 \sin u_{2}
\end{array}\right) \\
\quad-\left(\begin{array}{cc}
5.19 u_{1}-0.02 u_{1} \cos u_{1} \\
5.18 u_{2}-0.01 u_{2} \cos u_{2}
\end{array}\right)-\left(\begin{array}{cc}
0.1 & -0.03 \\
-0.03 & 0.1
\end{array}\right) f(u(t, x)) \\
-0.03 \quad 0.1
\end{array}\right) g(u(t-\tau(t), x))\right]\right\} d t+\sigma(u(t, x)) d w(t), \quad t \in\left[t_{k}, t_{k+1}\right) \\
u\left(t_{k}^{+}\right)=\left(\begin{array}{cc}
1.350 & 0 \\
0 & 1.350
\end{array}\right) u\left(t^{-}, x\right), \quad t=t_{k} \\
u\left(t_{0}+\theta, x\right)=\phi(\theta, x), \quad(\theta, x) \in[-0.65,0] \times \partial \Omega \\
\frac{\partial u_{i}(t, x)}{\partial v}=0, \quad(t, x) \in[-\tau,+\infty) \times \partial \Omega, \quad i=1,2
\end{gathered}
$$

where $\Omega=\left\{\left(x_{1}, x_{2}\right)^{T} \in R^{2} \mid-\sqrt{2} \leq x_{1}, x_{2} \leq \sqrt{2}\right\}$, and the corresponding matrices

$$
F=\left(\begin{array}{cc}
0.100 & 0 \\
0 & 0.100
\end{array}\right)=G, \quad Q=\left(\begin{array}{cc}
0.0001 & 0 \\
0 & 0.0001
\end{array}\right)
$$

We might as well assume that $t_{0}=0, t_{k}-t_{k-1}=0.525, \tau(t)=0.65, \tau^{\prime}(t) \leq \mu=0.99$ for all $t \geq t_{0}$, and

$$
\begin{gathered}
f(u)=g(u)=\left(\begin{array}{l}
\frac{\left|u_{1}+1\right|-\left|u_{1}-1\right|}{20} \\
\frac{\left|u_{2}+1\right|-\left|u_{2}-1\right|}{20}
\end{array}\right), \\
\phi(s, x)=\left(\begin{array}{c}
x(1-\cos (5 \pi x)) \cos ^{189}(x-0.25) e^{-100 s} \\
(1-x) \sin ^{2}(4 \pi x) \cos ^{201}(x-0.55) e^{-100 s}
\end{array}\right), \quad-0.65 \leq s \leq 0, \\
\underline{A}=\left(\begin{array}{ll}
1 & 0 \\
0 & 1
\end{array}\right), \quad \bar{A}=\left(\begin{array}{ll}
2 & 0 \\
0 & 2
\end{array}\right), \quad B=\left(\begin{array}{cc}
5.160 & 0 \\
0 & 5.160
\end{array}\right) .
\end{gathered}
$$

By way of MATLAB LMI Control Toolbox, we can solve the LMI condition in (C1) and get

$$
P_{1}=\left(\begin{array}{cc}
0.2392 & 0 \\
0 & 0.2392
\end{array}\right), \quad P_{2}=\left(\begin{array}{cc}
1.2291 & 0 \\
0 & 1.2291
\end{array}\right)
$$

Next, we will prove that such $P_{1}$ and $P_{2}$ make (C2) and (C3) hold. Indeed, by computing directly, we can obtain $a=0.01, b=0.0081$, and then (C2) holds. Moreover, we might as well 
assume $\delta=82$, and then we have $\lambda=0.0019, \rho=2.8235$, thus (C3) is satisfied. Now from Theorem 2.1 we can compute the convergence $9.3809 * 10^{-0.004}$.

\section{Conclusions}

In this paper, we investigate the influence of impulse, time-delays and diffusion behaviors on the stability of stochastic Cohen-Grossberg neural network (CGNN). The LMI conditions of stochastic exponential stability of impulsive CGNN with $p$-Laplace reaction-diffusion terms was given, and an illustrate example was also given to show the effectiveness of the obtained result. Besides, the result obtained in this paper is also valid to the Laplace reaction-diffusion (in the case of $p=2$ ) and has more computational efficiency due to the LMI approach even if $p=2$ (Remark 2.2).

\section{Acknowledgments}

This work was supported by the National Basic Research Program of China (2010CB732501), by Scientific Research Fund of Science Technology Department of Sichuan Province 2011JYZ010, and by Scientific Research Fund of Sichuan Provincial Education Department (11ZA172, 12ZB349).

\section{References}

[1] M. A. Cohen and S. Grossberg, "Absolute stability of global pattern formation and parallel memory storage by competitive neural networks," IEEE Transactions on Systems, Man, and Cybernetics, vol. 13, no. 5, pp. 815-826, 1983.

[2] K. Yuan and J. Cao, "An analysis of global asymptotic stability of delayed Cohen-Grossberg neural networks via nonsmooth analysis," IEEE Transactions on Circuits and Systems I, vol. 52, no. 9, pp. 18541861, 2005.

[3] S. Arik and Z. Orman, "Global stability analysis of Cohen-Grossberg neural networks with time varying delays," Physics Letters A, vol. 341, no. 5-6, pp. 410-421, 2005.

[4] J. Zhang, Y. Suda, and H. Komine, "Global exponential stability of Cohen-Grossberg neural networks with variable delays," Physics Letters A, vol. 338, no. 1, pp. 44-50, 2005.

[5] X. H. Zhang, S. L. Wu, and K. Li, "Delay-dependent exponential stability for impulsive CohenGrossberg neural networks with time-varying delays and reaction-diffusion terms," Communications in Nonlinear Science and Numerical Simulation, vol. 16, no. 3, pp. 1524-1532, 2011.

[6] M. Jiang, Y. Shen, and X. Liao, "Boundedness and global exponential stability for generalized CohenGrossberg neural networks with variable delay," Applied Mathematics and Computation, vol. 172, no. 1, pp. 379-393, 2006.

[7] Q. Song and J. Cao, "Stability analysis of Cohen-Grossberg neural network with both time-varying and continuously distributed delays," Journal of Computational and Applied Mathematics, vol. 197, no. 1, pp. 188-203, 2006.

[8] K. N. Wu and X. H. Ding, "Stability and stabilization of impulsive stochastic delay differential equations," Mathematical Problems in Engineering, vol. 2012, Article ID 176375, 16 pages, 2012.

[9] Y. F. Guo and F. L. Zhu, "New results on stability and stabilization of markovian jump systems with partly known transition probabilities," Mathematical Problems in Engineering, vol. 2012, Article ID 869842, 11 pages, 2012.

[10] K. Wang, Z. D. Teng, and H. J. Jiang, "Global exponential synchronization in delayed reactiondiffusion cellular neural networks with the Dirichlet boundary conditions," Mathematical and Computer Modelling, vol. 52, no. 1-2, pp. 12-24, 2010.

[11] J. Cao and J. Wang, "Global asymptotic stability of a general class of recurrent neural networks with time-varying delays," IEEE Transactions on Circuits and Systems I, vol. 50, no. 1, pp. 34-44, 2003. 
[12] P. Balasubramaniam and R. Rakkiyappan, "Delay-dependent robust stability analysis for Markovian jumping stochastic Cohen-Grossberg neural networks with discrete interval and distributed timevarying delays," Nonlinear Analysis: Hybrid Systems, vol. 3, no. 3, pp. 207-214, 2009.

[13] R. Rakkiyappan and P. Balasubramaniam, "Dynamic analysis of Markovian jumping impulsive stochastic Cohen-Grossberg neural networks with discrete interval and distributed time-varying delays," Nonlinear Analysis: Hybrid Systems, vol. 3, no. 4, pp. 408-417, 2009.

[14] M. Syed Ali and P. Balasubramaniam, "Robust stability of uncertain fuzzy Cohen-Grossberg BAM neural networks with time-varying delays," Expert Systems with Applications, vol. 36, no. 7, pp. 1058310588, 2009.

[15] X. Liang and L. S. Wang, "Exponential stability for a class of stochastic reaction-diffusion Hopfield neural networks with delays," Journal of Applied Mathematics, Article ID 693163, 12 pages, 2012.

[16] Y. T. Zhang, "Asymptotic stability of impulsive reaction-diffusion cellular neural networks with timevarying delays," Journal of Applied Mathematics, Article ID 501891, 17 pages, 2012.

[17] A. Salem, "Invariant regions and global existence of solutions for reaction-diffusion systems with a tridiagonal matrix of diffusion coefficients and nonhomogeneous boundary conditions," Journal of Applied Mathematics, Article ID 12375, 15 pages, 2007.

[18] P. Balasubramaniam and C. Vidhya, "Global asymptotic stability of stochastic BAM neural networks with distributed delays and reaction-diffusion terms," Journal of Computational and Applied Mathematics, vol. 234, no. 12, pp. 3458-3466, 2010.

[19] D. J. Higham and T. Sardar, "Existence and stability of fixed points for a discretised nonlinear reactiondiffusion equation with delay," Applied Numerical Mathematics, vol. 18, no. 1-3, pp. 155-173, 1995.

[20] R. F. Rao, S. M. Zhong, and X. R. Wang, "Stochastic stability criteria with LMI conditions for Markovian jumping impulsive BAM neural networks with mode-dependent time-varying delays and nonlinear reaction-diffusion," Communications in Nonlinear Science and Numerical Simulation. In press.

[21] D. Yue, S. F. Xu, and Y. Q. Liu, "A differential inequality with delay and impulse and its applications to the design of robust controllers," Control Theory \& Applications, vol. 16, no. 4, pp. 519-524, 1999.

[22] X. H. Wang, Q. Y. Guo, and D. Y. Xu, "Exponential p-stability of impulsive stochastic Cohen-Grossberg neural networks with mixed delays," Mathematics and Computers in Simulation, vol. 79, no. 5, pp. 16981710, 2009. 


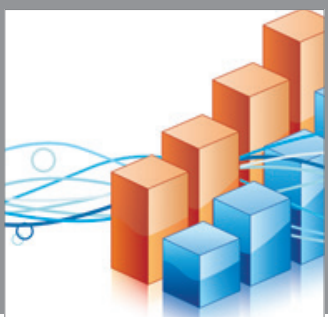

Advances in

Operations Research

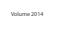

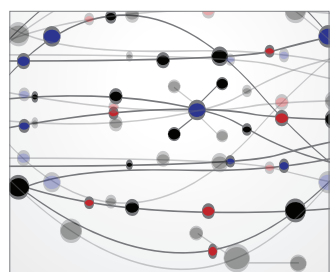

\section{The Scientific} World Journal
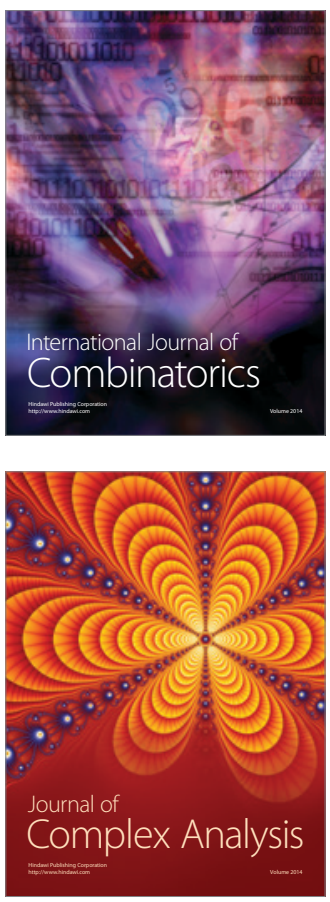

International Journal of

Mathematics and

Mathematical

Sciences
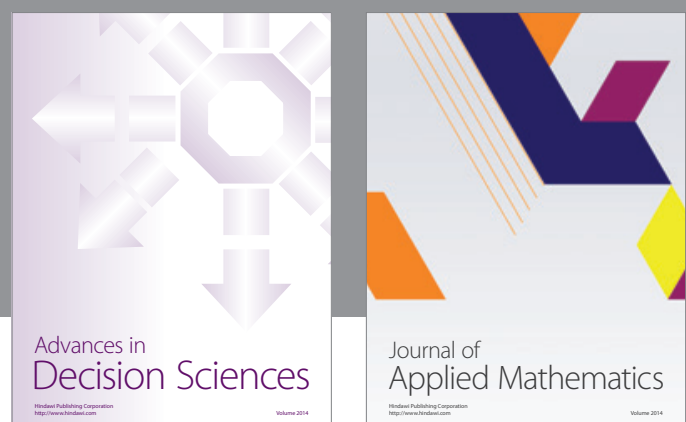

Journal of

Applied Mathematics
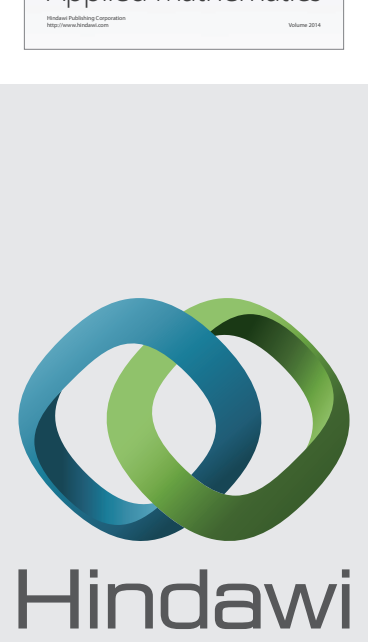

Submit your manuscripts at http://www.hindawi.com
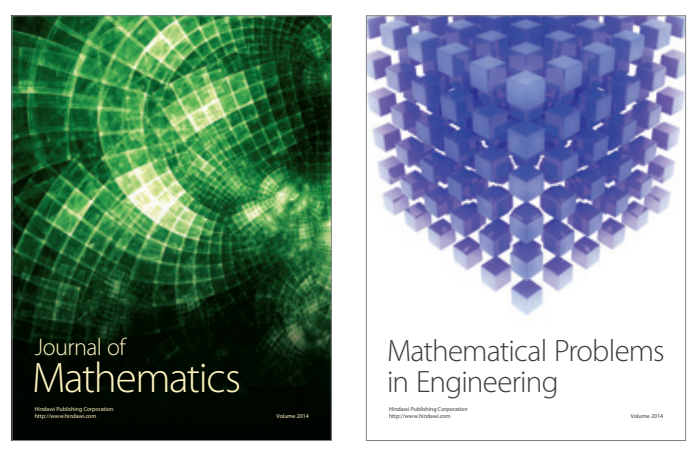

Mathematical Problems in Engineering
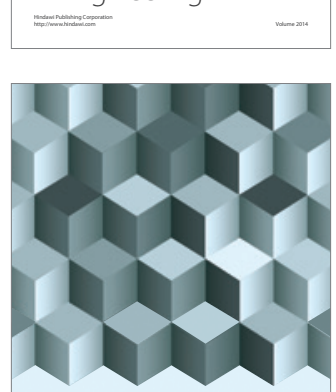

Journal of

Function Spaces
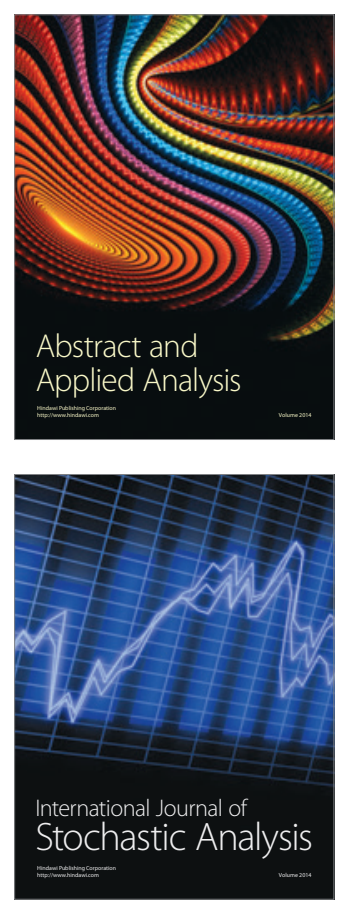

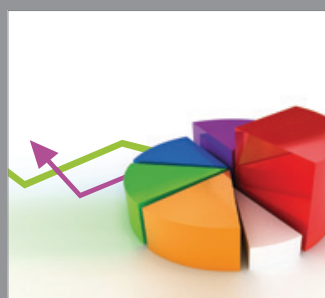

ournal of

Probability and Statistics

Promensencen
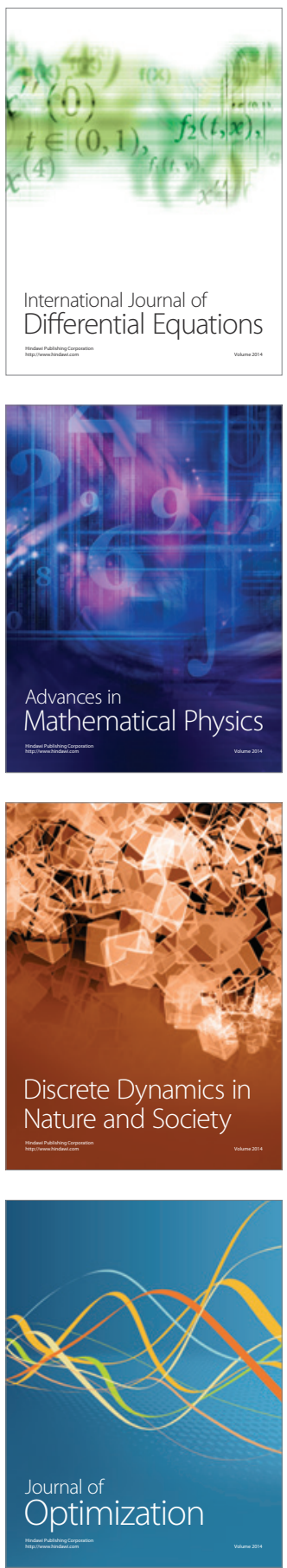